\title{
Special Section Guest Editorial: Get WIRED!
}

\author{
Zakya H. Kafafi ${ }^{\mathrm{a}, *}$ and Mónica Lira-Cantúb,c \\ aLehigh University, Department of Electrical and Computer Engineering, Bethlehem, \\ Pennsylvania, United States \\ ${ }^{\mathrm{b}}$ Catalan Institute of Nanoscience, Barcelona, Spain \\ ${ }^{c}$ Barcelona Institute of Science and Technology, Barcelona, Spain
}

This special section of Volume 10, Issue 4 of the Journal of Photonics for Energy (JPE) is partially based on papers presented at the inaugural conference on Women in Renewable Energy (WiRE) in 2019. This conference was held under the umbrella of the Organic Photonics + Electronics Symposium which is part of the SPIE annual Optics + Photonics meeting. The theme of the conference was key trends in renewable energy research developed and led by women scientists and engineers from around the world. WiRE included four technical sessions - Progress in Halide Perovskite Solar Cells, Solar Energy Utilization and Light Management, Harvesting Energy from Photovoltaics and Triboelectrics, and Energy Conversion and Storage - and a roundtable discussion, "The Role of Women Scientists in Renewable Energy." The papers in this special section represent an overview of a very small sample of ongoing research activities led by women researchers in academia worldwide.

Angela N. Fioretti from the École Polytechnique Fédérale de Lausanne in Switzerland, and Monica Morales-Masis from the University of Twente in the Netherlands have identified materials with mixed-anion chemistry and nonoxide materials as next-generation p-type transparent conducting materials (TCMs). ${ }^{1}$ Their theoretical studies outline recommendations for synthesizing these p-type TCMs and highlight remaining experimental barriers to be overcome.

Hima A. Kavuri from the University of Auckland in New Zealand and her collaborators from the MacDiarmid Institute for Advanced Materials and Nanotechnology in New Zealand have introduced a new generation of anode interlayers (AILs) for improving the efficiency and stability of organic photovoltaic devices. ${ }^{2}$ They used poly(vinyl pyrrolidone) (PVP) as an AIL modifier to alter molybdenum trioxide $\left(\mathrm{MoO}_{3}\right)$ and vanadium pentoxide $\left(\mathrm{V}_{2} \mathrm{O}_{5}\right)$. The PVP-metal oxide AILs improved the overall device quality, producing a nanotextured morphology with good optical properties and favorable chemical composition. Beneficial wetting properties for interfacial adhesion between the anode and the active layer were observed using contact angle measurements. Overall, devices with PVP-modified metal oxide AILs showed promising results with greater device stability compared to pure metal oxide AIL-based devices.

Bernice Mae Yu Jeco-Espaldon and her colleagues from the University of Tokyo in Japan and the University of Santo Tomas in the Philippines introduced a pathway to possibly homogenize and increase current collection in a limiting cell of a III-V multiple junction solar cell (MJSC) through the application of a perovskite quantum dot (PQD)-amorphous thermoplastic film. ${ }^{3}$ The PQD- cyclic olefin copolymer (COC) on a full III-V MJSC device successfully demonstrated current redistribution from the top cell and improved current homogeneity in the lower subcells - either by direct light re-emission from the PQD-COC layer or by consequently enhancing the luminescent coupling effect between adjacent lower bandgap sub-cells.

Researchers from California State University in Fullerton analyzed the performance of fixed topology and reconfigurable photovoltaic (PV) panel under partial shading conditions. ${ }^{4}$ Based on the PV characteristics of each topology, the CMOS-embedded PV panels outperformed other fixed topology-based PV panels. Reconfigurable PV panels provide a smart solution that can dynamically adjust to different configurations to outperform other fixed topologies in partial shading conditions.

Qing-Yun Chen and coauthors from Xi' an Jiaotong University in China studied temperature effect on green-synthesized $\mathrm{Co}_{3} \mathrm{O}_{4}$ nanoparticle photocatalysts for water splitting. ${ }^{5}$ The results

\footnotetext{
*Address all correspondence to Zakya H. Kafafi, kafafiz@gmail.com

(C) 2020 Society of Photo-Optical Instrumentation Engineers (SPIE)
} 
showed that, with the increase of ambient temperature, the evolution rate of hydrogen and oxygen was accelerated, and the atomic ratio of hydrogen to oxygen was close to 2:1. In addition, the $\mathrm{Co}_{3} \mathrm{O}_{4}$ photocatalyst had good stability. Their study provides an environmentally friendly, lowcost, and efficient method for the preparation of cobalt oxide photocatalysts with excellent performance.

We are grateful to all the authors for their contributions to this special section of the Journal of Photonics for Energy celebrating women working in the field of renewable energy. We hope that readers from diverse fields of science and engineering across the world will enjoy this small, representative collection of papers, which may serve as an inspiration for some to initiate and/or continue pursue research in renewable energy. Finally, we express our sincere thanks to all former and present editorial staff members of the Journal of Photonics for Energy for their hard work and support.

\section{References}

1. A. N. Fioretti and M. Morales-Masis, "Bridging the p-type transparent conductive materials gap: synthesis approaches for disperse valence band materials," J. Photonics Energy 10(4), 042002 (2020).

2. H. A. Kavuri et al., "Poly(vinyl pyrrolidone)-modified metal oxide anode interlayers for stable organic solar cells," J. Photonics Energy 10(4), 042003 (2020).

3. B. M. Y. Jeco-Espaldon et al., "Application of perovskite quantum dots in carrier redistribution in III-V multijunction solar cells with luminescent coupling effect," J. Photonics Energy 10(4), 042005 (2020).

4. R. V. Mahto, "Improving performance of photovoltaic panel by reconfigurability in partial shading condition," J. Photonics Energy 10(4), 042004 (2020).

5. Q.-Y. Chen, "Temperature effect on green-synthesized $\mathrm{Co} 3 \mathrm{O} 4$ nanoparticle as photocatalyst for overall water splitting," J. Photonics Energy 10(4), 042006 (2020).

Zakya H. Kafafi is a Distinguished Research Fellow at Lehigh University in Bethlehem, Pennsylvania. She was the Division Director of Materials Research at the National Science Foundation, and Section Head of Organic Optoelectronics at the Naval Research Laboratory where she pioneered studies on organic light-emitting diodes and solar cells. She is a Fellow of AAAS, MRS, OSA, and SPIE. She was the founding Editor-in-Chief of the Journal of Photonics for Energy and the inaugural Deputy Editor of Science Advances.

Mónica Lira-Cantú is Group Leader at the Nanostructured Materials for Photovoltaic Energy Group at the Catalan Institute of Nanoscience and Nanotechnology (www.icn2.cat), in Barcelona (Spain). Her research interests are the synthesis and application of nanostructured materials for next-generation solar cells: dye-sensitized, hybrid, organic and perovskite solar cells. She has more than 104 publications in scientific journals, 1 book, 9 book chapters, 9 patents, and h index of 46 (2020). 